\title{
Sil- \\ Segregación horizontal y vertical de género en el profesorado
}

\author{
JoRge FLETA-Asín \\ Área de Economía y Dirección de Empresas. Centro Univer- \\ sitario de la Defensa. Academia General Militar, Zaragoza.
} jorge.fleta@unizar.es

\section{FANG PAN}

Fac. DE Traducción e InTERPRETAción. Universidad Autónoma de Barcelona.

Centro de Lenguas Modernas. Universidad de Zaragoza. fpan@unizar.es

Resumen: En el presente trabajo se examina la segregación por género que existe en el profesorado en España durante el periodo 2004-2014. Para ello, en la introducción se justifica la importancia de la igualdad de género en las organizaciones. Posteriormente, en el apartado de literatura académica, se revisan los fundamentos sobre las causas que explican su existencia y se establece como hipótesis de trabajo que la discriminación

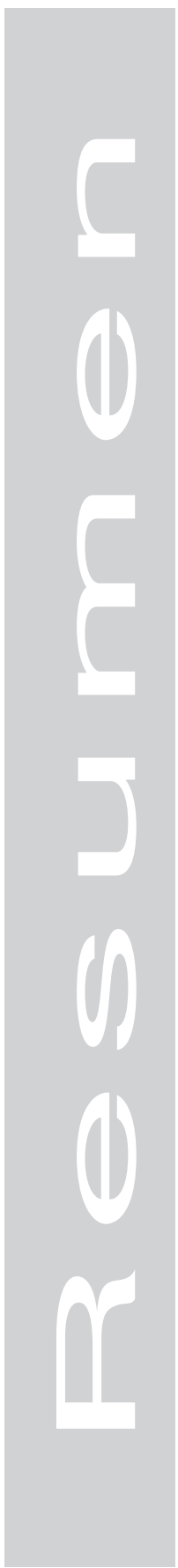


debería disminuir en los últimos años. A continuación, en el apartado de metodología y métodos se expone y calcula la segregación horizontal del profesorado entre los distintos niveles de educación a través del Índice de Disimilitud, así como la segregación vertical a través del Índice del Techo de Cristal. Los resultados revelan que continúan existiendo discriminaciones por género en el profesorado, si bien a lo largo del tiempo han disminuido tanto las diferencias horizontales como verticales. Además, el análisis muestra que las discriminaciones horizontales se han corregido menos que las dificultades para ascender una vez se decide el campo educativo en el que trabajar, por lo que la concienciación hacia las mujeres -frente a las medidas legislativas- resulta un elemento clave para alcanzar mayor igualdad en el ámbito educativo.

Palabras clave: Género, discriminación horizontal, discriminación vertical, Índice de Techo de Cristal, Índice de Disimilitud, Teoría de la Dominancia Social, Teoría del Rol Social.

\title{
Horizontal and Vertical Segregation of Gender in the Teaching Profession
}

\begin{abstract}
In this paper we examine gender segregation in the teaching profession in Spain between 2004 and 2014. With this in mind, the introduction justifies the importance of gender equality in organisations. Subsequently, in the section on academic literature, the fundamentals are reviewed concerning the causes that explain its existence, with the working hypothesis that discrimination should diminish over the years. The section on methodology and methods subsequently sets out and calculates horizontal segregation in the teaching profession at various levels of education using the Index of Dissimilarity, and also vertical segregation using the Glass-ceiling index. The results reveal that gender discrimination still exists in the teaching profession, even though both horizontal and vertical differences have decreased over the years. Furthermore, our analysis shows that horizontal discrimination has been corrected less frequently than the difficulties encountered to move up the ladder once the educational field of work has been decided upon. Hence, awareness of women - as opposed to legislative measures - turns
\end{abstract}


out to be a key element for reaching greater equality in the educational sector.

Keywords: Gender, horizontal discrimination, vertical discrimination, Glass-Ceiling Index, Index of Dissimilarity, Social Dominance Theory, Social Role Theory. 



\section{Segregación horizontal y vertical de género en el profesorado}

Jorge

Fleta-Asín

Recibido: 30/05/2016

Aceptado: 14/02/2017

Fang

Pan

Agradecimientos: Los autores quieren agradecer el apoyo del grupo de investigación CREVALOR (S17), financiado por el Gobierno de Aragón y fondos FEDER.

También se quiere agradecer el apoyo del Centro Universitario de la Defensa de Zaragoza y del Centro de Lenguas Modernas de la Universidad de Zaragoza.

\section{INTRODUCCIÓN}

En las últimas décadas existe un interés creciente hacia las cuestiones de género. Esta tendencia se consolidó a nivel mundial a partir de la Conferencia de Beijing (1995) de las Naciones Unidas, donde la declaración en favor del empoderamiento de la mujer y política mundial sobre igualdad de género se adoptó de forma unánime por 189 países.

En el caso de España, la deseabilidad en la igualdad entre hombres y mujeres queda reflejada principalmente en su Constitución (1978), en la Ley Orgánica 3/2007 de 22 de marzo y en los Planes Estratégicos de Igualdad de Oportunidades vigentes hasta la actualidad (el último del periodo 2014-2016).

Para analizar si los cambios impulsados se producen realmente se necesita información oportuna. Con este propósito a nivel nacional (p.e. Gobierno Británico o Gobierno de España) incorporan paulatinamente estadísticas desagregadas por sexos. Además, en los últimos años los organismos internacionales realizan esfuerzos en proporcionar información estadística sobre la disparidad de género comparada entre países (p.e. The Global 
Gender Gap Report 2015), publicaciones de libros (p.e. Mayoux y Hartl, 2009) y numerosos informes (p.e. Castaño et al., 2009; Grown et al., 2005).

Las fuentes de información, en cuanto a estadísticas y publicaciones, se revelan como necesarias para adecuar las políticas de igualdad y disminuir las brechas de género. Sin embargo, los indicadores que recogen las diferencias por sexo presentan los porcentajes de mujeres existentes en distintos ámbitos (por ejemplo porcentaje de mujeres directoras de centros educativos), sin considerar ese porcentaje respecto al total de mujeres que inicialmente se dedican a esos campos (por ejemplo proporción de mujeres que se dedican a la educación). Por ello, conviene no sólo distinguir las estadísticas en cuanto a diferencias por sexo, sino también utilizar indicadores más precisos para evaluar su diagnóstico y analizar las causas que las generan para poder incidir sobre las mismas.

Con este objetivo, en el siguiente apartado se revisan los fundamentos que explican la existencia de desigualdades de género en las organizaciones, estableciendo la hipótesis de que la segregación por género estaría disminuyendo en el profesorado en España. Posteriormente se explican la metodología, fuentes y resultados que contrastan la hipótesis propuesta mediante dos indicadores escasamente estudiados. Finalmente, en el último apartado, se establecen las conclusiones.

\section{LITERATURA ACADÉMICA SOBRE EL GÉNERO EN LAS ORGANIZACIONES}

El sexo de un individuo, el hecho de ser hombre o mujer, es un concepto genético definido por la biología (Holdcroft, 2007; Meier-Pesti y Penz, 2008).

Sin embargo el género es una construcción social porque comprende las tareas, funciones y roles atribuidos a las mujeres y los hombres tanto en la sociedad como en la vida pública y privada (Eagly y Wood, 2016). Por ello es habitual que el género se trate desde distintas disciplinas sociales como la psicología (Lyons, 1997; Rubio, 2016), sociología (Alberdi, 2005), filosofía (Leung, 2003), política (Tong, 2003; Leung, 2003; Rubio, 2016), 
economía (Dwyer et al., 2001; Luis-Carnicer et al., 2008; Kim et al., 2007) o antropología cultural (Hofstede y Hofstede, 2005).

A pesar de su diferenciación, el sexo aparece frecuentemente asociado al género en cuanto al hombre y a la mujer se les asocia una forma de comportamiento determinada (Holdcroft, 2007). Esto se debe a que la diferencia sexual biológica comprende las diferencias genéticas, hormonales y neuronales entre los distintos sexos. Así, estudios neurológicos establecerían que los dos hemisferios del cerebro son diferentes en el hombre y la mujer, determinando que la mujer tenga un proceso cognitivo diferente además de ser mejor en determinadas áreas del conocimiento, mientras que el hombre lo es en otras distintas (Kim et al., 2007; Dinç, 2008). O por ejemplo otros estudios sugieren que la testosterona como hormona esteroide principalmente masculina, se asociaría a determinados comportamientos como la agresividad y el riesgo (Meier-Pesti y Penz, 2008; Sibley y Harré, 2009). En base a estas diferencias biológicas podrían existir una serie de actitudes, juicios, formas de proceder y pensar diferentes entre el sexo masculino y femenino ante una misma situación (Kim et al 2007; Mclnerney et al., 2010).

Sin embargo, la Teoría del Rol Social cuestiona el determinismo biológico, defendiendo que la atribución de capacidades o características por sexo son presunciones que se cumplen porque la sociedad impone y espera unas pautas de conducta acordes a la categoría sexual (Eagly et al., 2000; Eagly y Wood, 2016). Desde esta perspectiva se sostiene que la personalidad atribuida al hombre y la mujer resulta un estereotipo y prejuicio de género (Rubio, 2016). Por ejemplo, esto se produce cuando al hombre se le supone más asertivo, independiente y racional, mientras que a la mujer se le cree más pasiva y emocional (Kray y Thompson, 2005; Agut y Martín, 2007). O cuando al hombre se le presume mejor en campos como las matemáticas, y a la mujer en relaciones sociales y la comunicación en general (Reilly et al., 2015; Caprile et al., 2008).

Discernir en el comportamiento de los individuos entre las predisposiciones genéticas y lo construido por interacciones sociales no es sencillo, ya que algunos rasgos vinculados al hombre y la mujer están asociados y mezclados con su proceso de socialización (Alberdi, 2005; Meece et al., 2006; Meier-Pesti y Penz, 2008; Sibley y Harré, 2009) (ver Tabla 1). 
Tabla 1. Dicotomía entre el sexo y el género

\begin{tabular}{|c|c|}
\hline Sexo & Género \\
\hline Genética & Psicológica \\
\hline Molecular & Entorno \\
\hline Celular & Social \\
\hline Fisiológica & Cultural \\
\hline
\end{tabular}

Fuente: Holdcroft, 2007.

A pesar de la complejidad para determinar las causas de estas diferencias, en general se acepta que desde la antigüedad la diferencia sexual biológica se canalizó y reforzó hacia la de géneros por la influencia de las sociedades patriarcales (Ferrer y Bosch, 2005; Eagly y Wood, 2016). Esta forma organizativa habrían prolongado la división sexual del trabajo de las sociedades cazadoras-recolectoras hasta la de géneros vigente en la actualidad (Eagly y Wood, 2016; Alberdi, 2005).

La Teoría de la Dominancia Social explicaría su persistencia y estacionariedad a lo largo del tiempo hasta el momento (Sidanius y Parto, 1999). Ésta sugiere que las sociedades se organizan en torno a una ordenación jerárquica entre los grupos que la constituyen, de forma que se minimiza el conflicto social o grupal gracias a las ideologías que justifican la desigualdad social inicial (Martínez et al., 2000). La continuación y legitimización de la diferencia de géneros se explicaría por aquellos grupos que ostentan el poder, en este caso los hombres, que deciden perpetuarse a través de sociedades patrilineales para seguir teniendo situaciones privilegiadas frente a las mujeres (Castillo y Montes, 2008).

El patriarcado reproduciría su sistema social a través de numerosos ámbitos que influyen en la forma de pensar de la sociedad, construyendo así la cosmovisión colectiva desde un punto de vista androcéntrico, y retroalimentando su dominancia sobre la mujer. Estos ámbitos abarcan medios tan diversos como el lenguaje a través del discurso hetero-normativo (Johnson, 2002; Rubio, 2016), la ley (Henderson et al., 1991), la literatura (Alberdi, 2005), la filosofía (Li, 2000; Leung, 2003), la religión (Elósegui, 2002; Rubio, 2016) o la visión sobre las ciencias (Miqueo et al., 
2003), transformando relaciones asimétricas de poder entre hombre-mujer en situaciones aparentemente lógicas y normalizadas.

Los medios instrumentalizados y sus argumentos se articularían a través de las distintas etapas del desarrollo vital del individuo comenzando por la propia familia (Davis, 2006), continuando en el centro de estudios (Ramaswami et al., 2010) y posterior entorno de trabajo (Agut y Martín, 2007), incluyendo su relación con el estado y las instituciones (Hofstede y Hofstede, 2005), donde en conjunto se procede a la construcción social y cultural del género (Alberdi, 2005; Caprile et al., 2008).

La interacción del discurso dominante desde distintos ámbitos y su repetición en estructuras organizativas cotidianas, construirían la lógica que justifica lo que se espera de la mujer y sus expectativas dentro de un universo simbólico que cobra sentido entre sus participantes. De este modo, el rol de la mujer se puede considerar como una respuesta a una expectativa figurada, proyectada desde un sistema social predeterminado, la sociedad patriarcal.

A pesar de la estrategia de normalización, las desigualdades terminan visibilizándose en discriminaciones horizontales y verticales (Charles, 2015; Agut y Martín, 2007). Por un lado, las segregaciones horizontales explicarían las diferencias existentes entre sexos en diferentes campos, disciplinas o áreas de trabajo de forma longitudinal (Miqueo et al., 2003; Agut y Martín, 2007). Mientras que las discriminaciones verticales se manifiestan transversalmente en las organizaciones jerárquicas, donde la mujer se encuentra escasamente representada en puestos altos de responsabilidad y salarios (Chi y Li, 2007; Neumark et al., 1996).

Las discriminaciones horizontales pueden generarse por las predisposiciones de la mujer hacia ciertos campos de estudio y trabajo, al renunciar voluntariamente a determinadas disciplinas en el proceso de construcción de su identidad de género (Caprile et al., 2008). La mujer verificaría así la Profecía Autocumplida, al compartir la creencia de que existen campos para los que es más apta, por lo que declinaría dedicarse a unos ámbitos concretos y predispondría su elección hacia otros para los que se cree más capacitada (Agut y Martín, 2007).

Esto parece ocurrir en algunas disciplinas como las matemáticas, física e ingeniería generalmente asociadas al hombre; 
mientras que la educación, psicología y ciencias de la salud se relacionan más con la mujer (Eisner y Harvey, 2009). En estudios postuniversitarios y trabajo académico también existirían estas diferencias. Concretamente en la sociología y las ciencias políticas la mujer aparece representada en mayor proporción que los hombres, mientras que en otros como la economía la mujer es una minoría (Beutel y Nelson, 2006).

A pesar de la sobre-representación de la mujer en algunos campos, los puestos más altos de la mayoría de las disciplinas no son ocupados por mujeres, lo que podría deberse a discriminaciones de tipo vertical que les impiden ascender (Guil et al 2004).

Estas discriminaciones verticales, pueden darse desde el núcleo familiar y desde la propia organización en la que trabaja. Desde el plano personal el hombre como cónyuge puede percibir que el desarrollo profesional de la mujer puede ser peligroso, porque compromete su contribución a la unidad familiar que forman ambos (Zarrow, 1988; Leung, 2003) y, desde la perspectiva de la organización, se puede percibir de forma análoga, que la mujer tenga una menor contribución a la empresa por sus cargas familiares (Agut y Martín, 2007).

Además, la Teoría del Escalón de Arriba expone que las personas que ocupan los órganos y puestos superiores en una organización son las que finalmente toman las decisiones (Dwyer et al., 2001), que en el caso de ser hombres podrían estar influidas por su visión androcéntrica (Agut y Martín, 2007). Precisamente, el hombre en general se encuentra mejor trabajando en grupos en los que hay más hombres por la similitud que presentan con ellos y la menor conflictividad que se deriva (Agut y Martín, 2007), además de que a las mujeres no se les considera adecuadas para determinadas tareas en las que se requiere autoridad y respeto (Luis-Carnicer et al 2008). Estas circunstancias frenarían que la mujer ocupase puestos de responsabilidad en las organizaciones, lo que se denomina techo de cristal o glass ceiling (Agut y Martín, 2007; Eisner y Harvey, 2009) ${ }^{1}$.

${ }^{1}$ El término techo de cristal se emplea tanto para explicar la dificultad de alcanzar los puestos de dirección más altos, como a las diferencias salariales que existen entre hombres y mujeres que ocupan una misma posición cuando ésta es alta. El suelo pegajoso o suelo de cemento (sticky floor), corresponde a las dificultades para ascender desde los puestos más bajos, así como a las diferencias salariales entre hombres y mujeres en escalas laborales bajas (Chi y Li, 2007; Kee, 2006). 
Las segregaciones por sexo están presentes en el ámbito educativo (Grossman y Grossman, 1994; McWirther, 1997). En concreto la Universidad presenta tanto discriminaciones de sexo horizontales en materias por estudiantes y profesores (Anguita et al., 2002; Puy et al., 2007), así como verticales en la promoción de su personal docente e investigador (Sánchez et al., 2005; Puy et al., 2007; Izquierdo et al., 2009).

Como se observa en la Figura 1, la combinación de ambos tipos de discriminaciones, verticales y horizontales, puede representarse en un eje bidimensional de coordenadas cartesianas. El eje horizontal o de abscisas representa, de izquierda a derecha, los ámbitos o campos agrupados de menor a mayor presencia de mujeres; mientras que el eje vertical o de ordenadas representa, de abajo a arriba dentro de cada ámbito, la presencia de menor a mayor grado de la mujer en la jerarquía.

\section{Figura 1. llustración de la segregación horizontal y vertical de género}

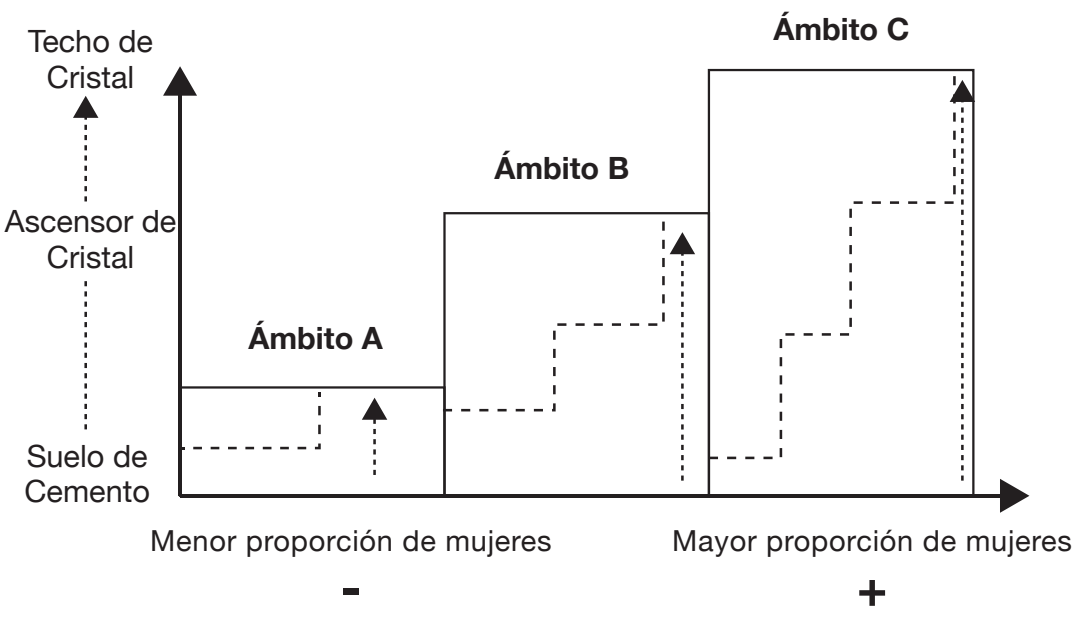

Fuente: Elaboración propia.

Así, en la Figura 1 se representa la proporción de mujeres en tres ámbitos o campos distintos: "A", "B" y "C". El eje horizontal representa los campos desde donde tiene menor presencia (" $A$ ") 
a mayor presencia ("C"). Dentro de cada ámbito se representan en líneas discontinuas un número hipotético de categorías profesionales, así como la dificultad de alcanzar cada una de ellas según la altura de cada escalón. El ámbito "A", presentaría dos categorías profesionales; mientras que el ámbito "C" tendría cuatro categorías. Esta foto fija permite visualizar de forma sintética la magnitud de la distribución desigual de la mujer en el conjunto de la sociedad, especialmente en la economía considerada formalmente como productiva por la menor incorporación de la mujer al mercado laboral.

A pesar de las diferencias expuestas, la tendencia a la igualdad es un valor que generalmente se propugna en las democracias liberales considerándose una condición deseable en la sociedad (Valcárcel et al., 2002; Aleixandre-Benavent et al., 2007), especialmente por cuestiones de justicia social (Aikman y Unterhalter, 2005). Concretamente sus fundamentos aparecen expuestos en la Declaración Universal de Derechos Humanos de las Naciones Unidas (1948) y Conferencia de Beijing (1995). El argumento básico sostiene que cuando la mujer no presenta las mismas posibilidades profesionales o, con el mismo cargo que un hombre se considera "inferior", se estarían cuestionando los principios de igualdad, capacidad y méritos. Además de las consideraciones legales y éticas, la ciencia económica expone que la desigualdad provoca el desaprovechamiento de capital humano, en este caso de mujeres, porque podrían desempeñar un papel más eficiente y productivo que algunos hombres sin desaprovechar así su talento (Vázquez et al., 1999; The Gender Gap, 2015).

Para corregir la situación, los gobiernos democráticos tratan de promover que la mujer presente las mismas oportunidades educativas y profesionales que se dan al hombre (Li, 2000). En este contexto, el ámbito educativo se revela como paradigma clave de cambio para impulsar la igualdad de género (Izquierdo et al., 2009). Una representación más equitativa de sexos en el profesorado normalizaría la imagen de igualdad entre hombre y mujer, ya que el periodo de formación es una de las fases socializadoras donde se reproducen esquemas de comportamiento de género que se toman como "correctos" (Caprile et al., 2008; Izquierdo et al., 2009; Lumadi y Shongwe, 2010). El proceso podría reforzarse especialmente en la Universidad porque es el lugar de 
formación previo al mercado laboral, centro de trabajo de docentes e investigadores, e institución que puede manifestar socialmente la igualdad (Garrison et al., 2010).

Los cambios que promueven las instituciones están alineados con el periodo histórico actual. En los últimos años con la Globalización y la Postmodernidad existe una desnaturalización de algunos rasgos dominantes en nuestro modo de vida entre los que se puede encontrar el patriarcado (Escudero, 1998) y concretamente la redefinición del papel de la mujer (Lyons, 1997; Borrás, 2007). Precisamente, existiría un cambio en el arquetipo masculino dominante que podría someterse a cambios históricos, sociales, étnicos o sexuales (Escudero, 2009).

Los cambios histórico-sociales y actitudinales en la consideración del género se ven coadyuvados por las políticas de igualdad encauzadas en los últimos años en España, que permiten pronosticar que se originarán cambios reales en la igualdad de las mujeres en todos los ámbitos, incluido el investigador (CSIC, 2007). En concreto, se considera que la Universidad española se abre progresivamente a las mujeres (Alemany et al 2006). Por ello, en base a estos argumentos se establece como Hipótesis que:

La segregación horizontal y vertical del profesorado disminuye en los últimos años en España.

\section{MATERIALES Y MÉTODOS}

Para analizar la segregación horizontal en los distintos niveles educativos se utiliza el Índice de Disimilitud (Wright y Ellis, 2000; Yalonetzky, 2012; Kalogrides y Loeb, 2013), mientras que para la segregación vertical dentro de las organizaciones se utiliza el Índice del Techo de Cristal (European Commission, 2011; Torres y Pau, 2011; Torres, 2012).

El Índice de Disimilitud (ID) es una medida demográfica de la uniformidad con la que dos grupos - en este caso hombres y mujeres - se distribuyen en campos que forman un área más grande - todas las categorías educativas estudiadas -. El resultado del índice se interpreta como el porcentaje de mujeres que tendría que reubicarse en diferentes áreas con el fin de producir 
una distribución que coincide con la que presenta en el conjunto inicial estudiado, acorde a la siguiente fórmula:

Índice de Disimilitud $(I D)=\frac{\sum_{i=1}^{N}\left|\frac{m_{i}}{M}-\frac{h_{i}}{H}\right|}{2}, 1 \geq I D \geq 0$

Siendo "i" cada una de las seis "N" categorías analizadas: "Centros E. Infantil y Primaria", "Centros de E. Secundaria, Bachillerato y FP", "Centros específicos de Educación Especial", "Enseñanzas Artísticas", "Enseñanzas de Idiomas" y "Educación Universitaria". "M" es la población total de mujeres para todas las áreas para las que se calcula el índice y " $m$ " la población total de cada una de las sub-áreas "i" analizadas. "H" es la población total de hombres en todas las áreas para las que se calcula el índice; y "h" la población total de hombres para cada uno de las categorías educativas "i” consideradas.

Por otro lado, el Índice del Techo de Cristal (ITC) es una medida utilizada para cuantificar la proporción del conjunto de mujeres que asciende a la máxima categoría de su profesión, en proporción a los hombres que ascienden a la misma posición. En este caso se calculan dos índices separados -uno para la enseñanza preuniversitaria y otro para la universitaria dado que presentan escalones profesionales diferentes- acorde a la siguiente fórmula:

Índice del Techo de Cristal $\left(\right.$ ITC) $=\frac{\frac{\sum_{i=1}^{N} \text { Mujeres }}{\text { Mujeres }} \text { Max.Categoria }}{\frac{\sum_{i=1}^{N} \text { Hombres }}{\text { Hombres }}} \quad, \quad \infty \geq I T C \geq 0$

Siendo "i" el número de personas existentes en cada uno de los escalones de la organización y "N" el número total de escalones profesionales existentes. En el caso de las enseñanzas no universitarias se consideran cuatro categorías: "profesorado", "secretaría", "jefatura de estudio" y "dirección de centro". En el caso de las enseñanzas universitarias son tres las figuras: "contratadas", "titulares" y "catedráticas". El subíndice "Max. Categoría" recoge el número total de personas que llegan a la 
cúspide organizativa según corresponda a hombres o mujeres: dirección del centro para las enseñanzas no universitarias y catedráticos para las universitarias. El índice alcanza valores entre 0 e infinito. Un valor de 1 indica que no existen dificultades en la promoción de hombres y mujeres. Valores por encima de 1 indican que las mujeres tienen un techo de cristal para ascender, mientras que valores por debajo de 1 indican que la mujer está sobre-representada. De forma análoga, se puede calcular el Índice del Techo de Cristal Progresivo (ITCP) para analizar la dificultad de ascender a una posición respecto a la inmediata inferior.

Para el estudio se analiza un intervalo de diez años, disponibles en el Ministerio de Educación, Cultura y Deporte en 2016, que corresponden al periodo educativo 2004-2014. Este periodo se analiza en tres cortes transversales simétricos: 2004-2005, 2007-2008, 2013-2014. Para el análisis se toma la totalidad del profesorado con las categorías más desagregadas que presentaban información sobre el número de mujeres. En el caso de las Universidades los datos se consideran exclusivamente para centros públicos, por estar incompleta la información del porcentaje de mujeres en instituciones privadas.

\section{RESULTADOS Y DISCUSIÓN}

\section{Resultados}

El profesorado del sistema educativo español parte de una sobre-representación de las mujeres respecto a los hombres, alcanzando una proporción ligeramente superior al $60 \%$ en el periodo 2004-2014 (Ministerio de Educación, Cultura y Deporte).

Dentro del ámbito educativo existen diferencias intra-grupo entre los distintos tipos de enseñanzas. De las categorías estudiadas en el último año académico disponible 2013-2014, la mayoría de las mujeres se concentran en educación infantil y primaria (84\%), educación especial $(81 \%)$, idiomas $(74 \%)$ y centros de ESO, Bachillerato y FP (56\%), presentando una menor proporción en enseñanzas artísticas (45\%) y universitarias (39\%) (Ver Anexo 1).

El Índice de Disimilitud permite calcular cuántas mujeres de las que se dedican a la enseñanza, deberían reubicarse entre los distintos tipos de educación para que no existiesen diferencias 
de género entre dichas categorías. Como se observa en la Tabla 2, el ID calculado en las cohortes 2004-2005, 2007-2008 y 2013-2014, muestra una tendencia decreciente, con unos valores de 0'46, 0’33 y 0'36 respectivamente. De modo que en el año 2013-2014, existiría un 36\% de mujeres que deberían redistribuirse entre diferentes enseñanzas para estar proporcionalmente agrupadas, una disminución de diez puntos porcentuales en los últimos diez años. Estos resultados corroboran la existencia de diferencias en la segregación horizontal y su disminución a lo largo del tiempo.

\section{Tabla 2. Índices de Disimilitud en centros educativos de Régimen General no universitarios y Universitarios. Todos los centros}

\begin{tabular}{|c|c|c|c|}
\cline { 2 - 4 } \multicolumn{1}{c|}{} & \multicolumn{3}{|c|}{ Años académicos } \\
\cline { 2 - 4 } & $2004-2005$ & $2008-2009$ & $2013-2014$ \\
\hline Índice de Disimilitud & 0,46 & 0,33 & 0,36 \\
\hline
\end{tabular}

Fuente: Elaboración propia a partir del Ministerio de Educación, Cultura y Deporte del Gobierno de España.

Una vez que el profesorado accede a su propio nivel de enseñanza, éste tiene distintas categorías para ascender. En el caso de la enseñanza no universitaria de régimen general y especial (que en el presente estudio comprende infantil y primaria; secundaria, bachillerato, módulos, FP; educación especial; educación artística; educación de idiomas) existen cuatro niveles de responsabilidad ordenados de menor a mayor rango: profesorado general, jefatura de estudios, secretaría y dirección del centro.

En el periodo 2006-2014, entre el 50 y $60 \%$ de las mujeres alcanzaron el puesto más alto como directoras de centro. La dificultad para alcanzar esta categoría puede observarse a través del Índice del Techo de Cristal (ver Tabla 3), que alcanza los valores de 1'35 (2006-2007), 1'22 (2008-2009) y 1'16 (20132014). Los valores por encima de 1 indican que existe una infrarepresentación de la mujer en los puestos más altos en relación al peso que tienen dentro de su régimen de enseñanza. Así, aunque el porcentaje de puestos directivos es mayoritariamente ocupado por mujeres (ver Anexo 2), según el ITC éstas muestran 
una proporción inferior a la que correspondería respecto a su peso inicial en el conjunto de estos campos.

\section{Tabla 3. Índices de Techos de Cristal en los centros educativos de régimen general no universitarios. Todos los centros.}

\begin{tabular}{|c|c|c|c|}
\cline { 2 - 4 } \multicolumn{1}{c|}{} & \multicolumn{3}{c|}{ Años académicos } \\
\cline { 2 - 4 } \multicolumn{1}{c|}{$\begin{array}{c}\text { Índice del Techo Cristal (ITC) } \\
\text { (Directoras respecto al Total Profesorado) }\end{array}$} & 1,35 & 1,22 & 1,16 \\
\hline $\begin{array}{c}\text { Índice del Techo Cristal Progresivo (ITCP) } \\
\text { (Secretarias de centro respecto al } \\
\text { Total Profesorado) }\end{array}$ & 1,29 & 1,18 & 1,13 \\
\hline $\begin{array}{c}\text { Índice del Techo Cristal Progresivo (ITCP) } \\
\text { (Jefas de estudio respecto al } \\
\text { Total Profesorado) }\end{array}$ & 1,24 & 1,16 & 1,13 \\
\hline
\end{tabular}

* En este caso se utiliza el año 2006-2007 porque es el más antiguo disponible.

${ }^{* *}$ El ITCP en estas enseñanzas se calcula respecto a todo el profesorado, ya que se puede acceder a cualquiera de sus escalones sin transitar previamente por el anterior.

Fuente: Elaboración propia a partir del Ministerio de Educación, Cultura y Deporte del Gobierno de España.

La dificultad para alcanzar progresivamente puede observarse a través del Índice del Techo de Cristal Progresivo (ITCP). En la Tabla 3 se observa que el indicador aumenta conforme se asciende más, mostrando que la dificultad para ascender de un escalón a otro es progresivamente mayor. Por ejemplo, en el año 2013-2014, para ser secretaria de centro el índice es de 1,13 , mientras que para llegar a directora es de 1,16². Además, se observa que la tendencia de ambos índices ITC e ITCP en estas categorías educativas es paulatinamente menor a lo largo del tiempo (2006-2014), alcanzando valores cercanos a la desaparición de las barreras (valores próximos a 1).

${ }^{2}$ El Ministerio de Educación, Cultura y Deporte del Gobierno de España no presenta directamente el número de mujeres en puestos de responsabilidad, si bien se puede calcular el ITC a partir del porcentaje de mujeres con la equivalencia matemática que se muestra en el Anexo 4. 
En el caso del profesorado universitario español, la presencia de la mujer incrementó ligeramente desde el 35\% en 2004, hasta el 39\% en 2014 (ver Anexo 3). Un aumento de cuatro puntos porcentuales en un intervalo de diez años. La segregación vertical también está presente en estos cuerpos docentes. En el último año analizado (2013-2014), de los 10.562 catedráticos universitarios en la Universidad pública, el $20 \%$ eran mujeres. Una proporción inferior al peso que tiene la mujer dentro del profesorado universitario ya que el Índice del Techo de Cristal para alcanzar una plaza de catedrática presenta valores superiores a 1 (ver Tabla 4), si bien disminuyen a lo largo del tiempo alcanzando valores de 2,45 (2004-2005), 2,33 (2008-2009) y 1,89 (2013-2014).

\section{Tabla 4. Índices de Techos de Cristal en la Universidad pública en España}

\begin{tabular}{|c|c|c|c|}
\cline { 2 - 4 } \multicolumn{1}{c|}{} & \multicolumn{3}{c|}{ Años académicos } \\
\cline { 2 - 4 } \multicolumn{1}{c|}{$\begin{array}{c}\text { Índice del Techo de Cristal } \\
\text { (Catedráticas respecto al Total Profesorado) }\end{array}$} & 2,45 & 2,33 & 1,89 \\
\hline $\begin{array}{c}\text { Índice del Techo de Cristal Progresivo } \\
\text { (Catedráticas respecto al Total Titulares) }\end{array}$ & 2,24 & 2,10 & 1,68 \\
\hline $\begin{array}{c}\text { Índice del Techo de Cristal Progresivo } \\
\text { (Titulares respecto al Total Contratados) }\end{array}$ & 1,01 & 1,02 & 1,05 \\
\hline
\end{tabular}

Fuente: Elaboración propia a partir del Ministerio de Educación, Cultura y Deporte del Gobierno de España.

Sin embargo, en la Universidad existen dos diferencias en la segregación vertical que difieren respecto al sistema educativo preuniversitario. La primera es que en la Universidad pública el techo de cristal es más elevado que en la educación preuniversitaria. Así, en el año 2013-2014 para ser directora de centro el ITC era de 1,16 (ver Tabla 3), mientras que para ser catedrática era de 1,89 (ver Tabla 4). Esto quiere decir que para una mujer es más difícil ascender al nivel más alto en la Universidad que en niveles educativos previos. La segunda diferencia es que a pesar de la mayor dificultad en la Universidad, apenas existe un techo de cristal para 
ascender desde la categoría de contratada a titular, manteniendo valores cercanos a 1 , e incluso ligeramente crecientes durante todo el periodo $2004-2014$ (con valores de 1,$01 ; 1,02$ y 1,05).

\section{CONCLUSIONES}

Los resultados del presente trabajo revelan la existencia tanto de segregación horizontal como vertical del profesorado en España. Los hallazgos son acordes a la literatura académica que argumenta su presencia en las organizaciones. Como se establecía en la hipótesis formulada, ambos tipos de discriminación han disminuido a lo largo del periodo 2004-2014, confirmándose una convergencia gradual de género en campos educativos y escalas profesionales.

Los hallazgos contradicen algunas líneas argumentales que sostienen que el cambio hacia la igualdad podría estar realizándose de forma más retórica que sustantiva (Leung, 2003). Estos argumentos mantienen que los cambios aparentes se articularían instrumentalmente por parte del patriarcado a través de discursos y declaraciones que finalmente no se producirían. El grupo dominante acompañaría su retórica de cambio con la existencia de intentos simbólicos por alcanzar la paridad a través del tokenismo, o inclusiones de las mujeres como minoría para normalizar de forma aparente su situación (Beutel y Nelson, 2006). Por este motivo, en ocasiones la mujer creería que los problemas de discriminación se daban en generaciones anteriores pero no en la suya, cuando esto podría no ser así (Miqueo et al., 2003). Aunque se cuestiona si el cambio hacia la igualdad se estaría realmente materializando (Valcárcel et al., 2002), los resultados confirman que en el ámbito educativo español sí se está produciendo.

Otro de los resultados del trabajo es la existencia de menor segregación vertical en las escalas profesionales más bajas, aumentando progresivamente la dificultad para ascender en las organizaciones. Esto es común tanto en la enseñanza preuniversitaria como universitaria y apoya la literatura existente de que los escalones, o ascensor de cristal, son progresivamente de mayor tamaño. También cabe destacar que existen mayores segregaciones verticales en la Universidad que en la enseñanza preuniversitaria y que, a e pesar de lo que la teoría predice, el techo de 
cristal apenas existiría para ascender en algunas escalas inferiores, como la de contratado doctor a titular en la Universidad.

No obstante, estos resultados deben analizarse con prudencia. Por una parte porque la interpretación de los datos requiere un análisis del contexto, ya que el sector de la educación está altamente feminizado, por lo que la dificultad para ascender incluso en condiciones de partida donde están sobrerrepresentadas, constituye una discriminación mucho mayor. Por otro lado, porque la mejora en los indicadores podría no mostrar "desigualdades ocultas". Las cargas familiares de la mujer podrían provocar que internalizasen los costes profesionales en la unidad familiar, renunciando ellas mismas a ascender profesionalmente. En casos extremos, la mujer renunciaría a su desarrollo personal para ascender corrigiendo así la desigualdad numérica del índice, pero sin reflejar el mayor esfuerzo y sacrificio de la mujer. Además, incluso cuando la mujer alcanza las mismas posiciones que el hombre, a menudo se percibe que el hombre con la misma posición presenta mayores competencias y tiene mayor autoridad (Ramaswami et al., 2010), por lo que el mismo puesto no ofrecería el mismo reconocimiento a pesar de alcanzarse.

Por último, conviene resaltar que los índices calculados son sensibles al número de campos y al nivel de categorías profesionales estudiadas. En todo caso, los índices que tienen en cuenta la proporción inicial de mujeres respecto al porcentaje posterior que ocupan en sus escalas profesionales, resultan más indicados que estadísticas desagregadas que no valoran el peso inicial de la mujer en un ámbito de estudio.

\section{BIBLIOGRAFÍA}

Agut, S. y Martín, P. (2007). Factores que dificultan el acceso de las mujeres a puestos de responsabilidad: una revisión teórica. Apuntes de Psicología, 25(2), 201-214.

Aikman, S. y Unterhalter, E. (Eds.) (2005). Beyond access: Transforming policy and practice for gender equality in education. Oxfam. 
Alberdi, I. (2005). Cómo reconocer y cómo erradicar la violencia contra las mujeres. F. C. de Pensions (Ed.). Fundación" la Caixa".

Aleixandre, R. González, G. Alonso, A. Castellano, M. y Valderrama, J.C. (2007). Valoración de la paridad en la autoría de los artículos publicados en la Revista Enfermedades Infecciosas y Microbiología Clínica durante el quinquenio 20012005. Enfermedades Infecciosas y Microbiología Clínica, 25(10), 619-626.

Alemany Gómez, C., Díaz Martínez, C., \& Thonberg, E. (2006). Los últimos bastiones ¿Por qué las mujeres están ausentes de los puestos de mayor responsabilidad de la docencia y de la gestión de la enseñanza superior? Centre dEstudis Dona i Societat (CEDIS). Madrid: Instituto de la Mujer, España.

Anguita, R. Alario, M. Alario, M.T. Alario, A.I. García, C. Santo Tomás, M. del Val, M.I. y Vargas, M.A. (2002). Las mujeres en la Universidad de Valladolid: aspiraciones y realidad profesional. Madrid: Instituto de la Mujer, Ministerio de Igualdad.

Beutel, A.M. y Nelson, D.J. (2006). The gender and race-ethnicity of faculty in top social science research departments. The Social Science Journal, 43, 111-125.

Borrás, V. (2007). Las desigualdades en el consumo a través del género. Revista Española de Sociología, 8, 139-156.

Caprile, M. Vallès, N. Potrony, J. Crèixams, C. y Arasanz, J. (2008). El sesgo de género en el sistema educativo. Su repercusión en las áreas de Matemáticas y Tecnología en Secundaria. Madrid: Instituto de la Mujer, Ministerio de Igualdad.

Castaño, C., Laffarga, J., Iglesias, C., De Fuentes, P., Martín, J., Llorente, R., Charlo, M.J., Giner, Y., Vázquez, S., Núñez, M. y Martínez, J.L. (2009). Mujeres y poder empresarial en España. Madrid: Instituto de la Mujer, Ministerio de Igualdad, Gobierno de España, Madrid.

Castillo, M.R. y Montes, B. (2008). Aportaciones de la Teoría de la Dominancia Social al análisis de la discriminación de género. Iniciación a la Investigación, 3(1), 1-6.

Centro Superior de Investigaciones Científicas (2007). Plan de igualdad de género en la carrera científica de la agencia estatal 
CSIC (2007-2009). Madrid: Comisión de Mujeres y Ciencia del CSIC.

Charles, M. (2015). Sex Segregation. The Wiley-Blackwell Encyclopedia of Globalization. John Wiley \& Sons, Ltd.

Chi, W. y Li, B. (2007). Glass ceiling or sticky floor? Examining the gender earnings differential across the earnings distribution in urban China 1987-2004. Journal of Comparative Economics, 36, 243-263.

Conferencia de Beijing (1995). Resolución de las Naciones Unidas. Recuperado de http://www.un.org/womenwatch/daw/ beijing/pdf/BDPfA\%20S.pdf

Davis, S.N. (2006). Gender ideology construction from adolescence to young adulthood. Social Science Research, 35, 1021-1041.

Dinç, P. (2008). Gender (in)difference in private offices: A holistic approach for assessing satisfaction and personalization. Journal of Environmental Psychology, 29, 53-62.

Dwyer, S. Richard, O.C. y Chadwick, K. (2003). Gender diversity in management and firm performance: the influence of growth orientation and organizational culture. Journal of Business Research, 56, 1009-1019.

Eagly, A. H. y Wood, W. (2016). Social Role Theory of Sex Differences. The Wiley Blackwell Encyclopedia of Gender and Sexuality Studies. 1-3.

Eagly, A. H., Wood, W., y Diekman, A. B. (2000). Social role theory of sex differences and similarities: A current appraisal. The Developmental Social Psychology of Gender, 123-174.

Eisner, S.P. y Harvey, M.E. (2009). C-Change? Generation Y and the Glass Ceiling, S.A.M. Advanced Management Journal, 74(1), 13-28.

Elósegui, M. (2002). Mujeres y fundamentalismo. Aequalitas: Revista Jurídica de Igualdad de Oportunidades entre Mujeres y Hombres, (10), 9-13.

Escudero, M. (1999). El sueño de la razón produce monstruos en el silencio de los corderos de Jonathan Demme. Boletín del Museo e Instituto Camón Aznar, 77, 67-80. 
Escudero, M. (2009). La retórica ambivalente de la performance drag king: estereotipos y parodia de la masculinidad en un contexto anglosajón. Arte y Políticas de Identidad, (1), 49-64.

European Union (2011). Innovation Union Competitiveness Report 2011. Methodological Annex. Recuperado de https:// ec.europa.eu/research/innovation-union/pdf/competitiveness-report/2011/iuc2011-methodological-annex.pdf

Ferrer, V.A. y Bosch, E. (2005). Introduciendo la perspectiva de género en la investigación psicológica sobre violencia de género. Anales de Psicología, 21(1), 1-10.

Garrison, D.R. Cleveland-Innes, M. y Fung, T.S. (2010). Exploring causal relationships among teaching, cognitive and social presence: student perceptions of the community of inquiry framework. Internet and Higher Education, 13, 31-36.

Grossman, H., y Grossman, S. H. (1994). Gender issues in education. Allyn and Bacon, A Division of Simon \& Schuster, Inc., 160 Gould Street, Needham Heights, MA 02194.

Grown, C. Gupta, G.R. y Kes, A. (2005). Taking action: achieving tender equality and empowering women. London: United Nations, Millennium Project.

Guil, A., Jiménez, Y., Arriaga, M., Vicente, G., Ramírez, D., González, C. y Gonzales, B. (2004). Techos de cristal en la Universidad Hispalense. Madrid: Instituto de la Mujer, Ministerio de Igualdad.

Henderson, L., Eisenstein, Z. R., MacKinnon, C. A., y Smart, C. (1991). Law's patriarchy.

Hofstede, G. y Hofstede, G.J. (2005). Cultures and Organizations, software of the mind. United Kingdom: McGraw-Hill.

Holdcroft, A. (2007). Integrating the dimensions of sex and gender into basic life sciences research: methodological and ethical issues. Gender Medicine, 4, 564-574.

Izquierdo, M. J., Cruz, S., Cuccu, M., Duarte, L., Jiménez, E. y Santero, V. (2009). Percepción de los factores que intervienen en la evolución de la trayectoria académica: una perspectiva de género. Madrid: Instituto de la Mujer, Ministerio de Igualdad.

Johnson, C. (2002). Heteronormative citizenship and the politics of passing. Sexualities, 5(3), 317-336. 
Kalogrides, D., y Loeb, S. (2013). Different teachers, different peers the magnitude of student sorting within schools. Educational Researcher, 42(6), 304-316.

$\mathrm{Kee}, \mathrm{H}$. J. (2006). Glass ceiling or sticky floor? Exploring the Australian gender pay gap. Economic Record, 82(259), 408-427.

Kim, D.Y., Lehto, X.Y. y Morrison, A.M. (2007). Gender differences in online travel information search: Implications for marketing communications on Internet. Tourism Management, 28, 423-433.

Kray, L.J. y Thompson, L. (2005). Gender stereotypes and negotiation performance: an examination of theory and research. Research in Organizational Behavior, 26, 103-182.

Leung, A. S. M. (2003). Feminism in transition: Chinese culture, ideology and the development of the women's movement in China. Asia Pacific Journal of Management, 20(3), 359-374.

Ley Orgánica 3/2007 de 22 de Marzo. Boletín Oficial de España. Recuperado de https://www.boe.es/boe/dias/2007/03/23/ pdfs/A12611-12645.pdf

Li, C. (2000). The sage and the second sex: Confucianism, ethics, and gender. Open Court Publishing.

Luis-Carnicer, P., Martínez-Sánchez, A., Pérez-Pérez, M. y VelaJiménez, M.J. (2008). Gender diversity in management: curvilinear relationships to reconcile findings. Gender in Management: An International Journal, 23(8) 583-597.

Lumadi, M.W. y Shongwe, S.S. (2010). The need for training gender sensitive teachers: addressing education challenges for gender sensitive national development. Contemporary Issues in Education Research, 3(3) 41-49.

Lyons, D. (1997). The feminine in the foundations of organizational psychology. The Journal of Applied Behavioral Science, 33(1) 7-26.

Martínez, C., Paterna, C., Rosa, A.I. y Angosto, J. (2000). El principio de jerarquía social como explicación del prejuicio y el rechazo a la acción positiva. Psicología Política, 2, 55-71.

Mayoux, L. y Hartl, M. (2009). Gender and rural microfinance: Reaching and empowering women. Italy: International Fund for Agricultural Development. 
Mclnerney, M.L., Mader, D.D. y Mader, F.H. (2010). Gender differences in responses to hypothetical business ethical dilemmas by business undergraduates. Journal of Diversity Management, 5(9) 137-41.

McWhirter, E. H. (1997). Perceived barriers to education and career: Ethnic and gender differences. Journal of Vocational Behavior, 50(1), 124-140.

Meece, J. L., Glienke, B. B., y Burg, S. (2006). Gender and motivation. Journal of School Psychology, 44(5), 351-373.

Meier-Pesti, K. y Penz, E. (2008). Sex or gender? Expanding the sex-based view by introducing masculinity and femininity as predictors of financial risk taking. Journal of Economic Psychology, 29, 180-196.

Miqueo, C., Barral, M.J., Delgado, I., Fernández, T. y Magallón, C. (2003). Del análisis crítico a la autoridad femenina en la ciencia. Feminismo/s, 1, 195-216.

Neumark, D., Bank, R.J. y Van Nort, K.D. (1996). Sex discrimination in restaurant hiring: an audit study. The Quarterly Journal of Economics, 111(3), 915-941.

Puy, A., Barrera, M.C., González, R., Hernández, J.M., Santana, J.D., Díaz, M., García, M.J. y Morales, C. (2007). Barreras a la promoción de las mujeres a puestos de alto estatus en la Universidad. Madrid: Instituto de la Mujer, Ministerio de Igualdad.

Ramaswami, A., Dreher, G. F., Bretz, R. y Wiethoff, C. (2010). Gender, mentoring, and career success: the importance of organizational context. Personnel Psychology, 63(2), 385-405.

Reilly, D., Neumann, D. L., \& Andrews, G. (2015). Sex Differences in Mathematics and Science Achievement: A Meta-Analysis of National Assessment of Educational Progress Assessments. Journal of Educational Psychology, 107(3), 645-662.

Rubio, A.V. (2016). Cuando nos prohibieron ser mujeres... y os persiguieron por ser hombres. Madrid: Editorial La Factoría.

Sánchez, M.R., López, J., Mayor, C., Murillo, P. y Hernández, E. (2005). La mujer en la dirección y gestión de las organizaciones universitarias: problemática, estilos de liderazgo y contribución al desarrollo institucional. Madrid: Instituto de la Mujer, Ministerio de Igualdad. 
Schwab, K. Samans, R. Zahidi, S. Bekhouche, Y. Padilla, P. y Ratcheva, V. (2015). The Global Gender Gap Report 2015. World Economic Forum, Geneva.

Sibley, C.G. y Harré, N. (2009). A gender role socialization model of explicit and implicit biases in driving self-enhancement. Transportation Research, 12, 452-461.

Sidanius, J. y Pratto, F. (1999). Social dominance: an intergroup theory of social hierarchy and oppression. Cambridge University Press.

Tong, J. (2003). The gender gap in political culture and participation in China. Communist and Post-Communist Studies, 36, 131150.

Torres, O. (2012). Science and Gender Indicators: A Critical Review. International Journal of Gender, Science and Technology, 4(1), 24-47.

Torres, O. y Pau, B. (2011). "Techo de cristal" y "suelo pegajoso": La situación de la mujer en los sistemas alemán y español de ciencia y tecnología. Revista Iberoamericana de Ciencia, Tecnología y Sociedad, 6(18), 35-39.

Valcárcel, A. Miyares, A. y Herrera, A. (2002). Paridad II. Madrid: Instituto de la Mujer, Ministerio de Igualdad.

Vázquez, A. Manassero, M.A. Fernández, M.C. Picornell, C. Serra, B. y Erice, C. (1999). Los estereotipos de género en el currículo científico y tecnológico de secundaria. Actitudes y preferencias de alumnado y profesorado. Madrid: Instituto de la Mujer, Ministerio de Igualdad.

Wright, R. y Ellis, M. (2000). The Ethnic and Gender Division of Labor Compared Among Immigrants to Los Angeles. International Journal of Urban and Regional Research, 24(3), 583-600.

Yalonetzky, G. (2012). A dissimilarity index of multidimensional inequality of opportunity. The Journal of Economic Inequality, 10(3), 343-373.

Zarrow, P. (1988). He Zhen and anarcho-feminism in China. The Journal of Asian Studies, 47(4), 796-813. 


\section{ANEXOS}

\section{Anexo 1. Distribución del profesorado por sexo y tipo de centro en España 2013-2014.}

\begin{tabular}{|c|c|c|c|c|c|}
\cline { 2 - 6 } \multicolumn{1}{c|}{} & Tipo de estudios & Mujeres & Hombres & Mujeres (\%) & Total \\
\hline \multirow{2}{*}{$\begin{array}{c}\text { EE. RÉGIMEN } \\
\text { GENERAL } \\
\text { (no universitaria) }\end{array}$} & $\begin{array}{c}\text { Centros } \\
\text { E. Infantil y primaria }\end{array}$ & 235.394 & 44.698 & 84,04 & 280.092 \\
\cline { 2 - 6 } & $\begin{array}{c}\text { Centros ESO y/o } \\
\text { Bach. y/o FP }\end{array}$ & 125.962 & 96.386 & 56,65 & 222.348 \\
\cline { 2 - 6 } & $\begin{array}{c}\text { Centros específi- } \\
\text { cos E. Especial }\end{array}$ & 6.167 & 1.377 & 81,75 & 7.544 \\
\hline \multicolumn{2}{|c|}{ E. UNIVERSITARIA } & 45.912 & 69.159 & 39,90 & 115.071 \\
\hline \multirow{2}{*}{$\begin{array}{c}\text { EE. RÉGIMEN } \\
\text { ESPECIAL }\end{array}$} & EE. Artísticas * & 13.898 & 16.863 & 45,18 & 30.761 \\
\cline { 2 - 6 } & E. de Idiomas & 4.182 & 1.432 & 74,49 & 5.614 \\
\hline \multicolumn{2}{|c|}{ TOTAL } & 431.515 & 229.915 & 65,24 & 661.430 \\
\hline
\end{tabular}

* Las EE. Artísticas incluyen: Escuelas de Arte y C. Estudios Sup. Artes Plásticas y Diseño; Centros de EE. de la Música; Centros de EE. de la Danza; Escuelas de EE. de la Música y/o Danza; Escuelas de Arte Dramático.

Fuente: Elaboración propia a partir del Ministerio de Educación, Cultura y Deporte del Gobierno de España.

\section{Anexo 2. Porcentaje de mujeres en los equipos directivos de los centros educativos de régimen general no universitarios en España.}

\begin{tabular}{|c|c|c|c|}
\cline { 2 - 4 } \multicolumn{1}{c|}{} & \multicolumn{3}{c|}{ Años académicos } \\
\hline Directoras de centro & $\mathbf{2 0 0 6 - 2 0 0 7 *}$ & $\mathbf{2 0 0 8 - 2 0 0 9}$ & $\mathbf{2 0 1 3 - 2 0 1 4}$ \\
\hline Secretarias de centro & $50,30 \%$ & $56,70 \%$ & $61,80 \%$ \\
\hline Jefas de estudio & $52,40 \%$ & $58,70 \%$ & $63,30 \%$ \\
\hline Mujeres respecto a total hombres & $54,60 \%$ & $59,60 \%$ & $63,30 \%$ \\
\hline
\end{tabular}

* En este caso se utiliza el año 2006-2007 porque es el más antiguo disponible.

Fuente: Elaboración propia a partir del Ministerio de Educación, Cultura y Deporte del Gobierno de España. 
Anexo 3. Personal docente e investigador en la Universidad pública por sexo y categoría en España 2013-2014.

\begin{tabular}{|c|c|c|c|c|}
\cline { 2 - 5 } \multicolumn{1}{c|}{} & Mujeres & Hombres & Mujeres (\%) & Total \\
\hline Universidades públicas & 39.392 & 60.626 & $39,38 \%$ & 100.018 \\
\hline Funcionarios & 16.650 & 30.425 & $35,37 \%$ & 47.075 \\
\hline Catedráticos de Universidad (CU) & 2.182 & 8.380 & $20,66 \%$ & 10.562 \\
\hline Titulares de Universidad (TU) & 11.793 & 17.897 & $39,72 \%$ & 29.690 \\
\hline Contratados & 20.076 & 26.566 & $43,04 \%$ & 46.642 \\
\hline
\end{tabular}

Fuente: Elaboración propia a partir del Ministerio de Educación, Cultura y Deporte.

\section{Anexo 4. Índice del Techo de Cristal (ITC).}

Cálculo del ITC a partir del porcentaje total de mujeres y el porcentaje de su categoría ocupacional máxima:

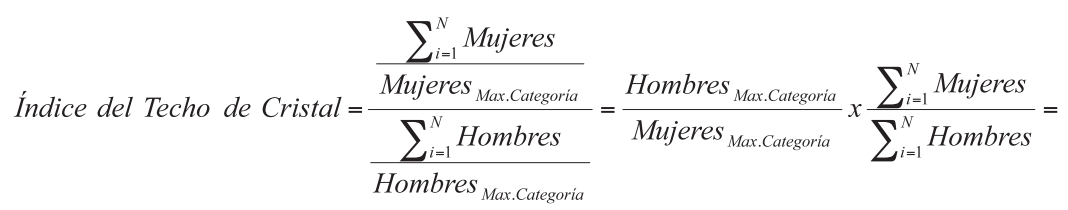
$=(\text { Porcentaje de Mujeres Max.Categoria })^{-1} x$ (Porcentaje de Hombres Max.Categoria $)$ 